\title{
Cognitive Radio Technology Based Spectrum Allocation for D2d-U Communication
}

\section{T.Veeramakali}

\begin{abstract}
In this paper, a heterogeneous network consists of Device to Device (D2D) communication users and Cognitive Radio (CR) network users are considered. D2D users can communicate through licensed band or unlicensed band. Unlicensed band accessing D2D users are called as D2D-U users. CR users are using the unlicensed band for data transmission and they have the capability to access the licensed band when it is not in use. This proposed system is considering D2D-U users and Wi-Fi users in a local geographical area and applying scheduling technique for both to access the licensed band without affecting the licensed user's transmission. The proposed system evaluated the data sum rate against number of user. The simulation results are generated and discussed.

Keywords: D2D-U,CR, white space, Spectrum
\end{abstract}

\section{INTRODUCTION}

In today's wireless world, the vast growth in innovation of wireless device and applications are the main reason for the spectrum scarcity. Two main technologies like CR and D2D are introduced to overcome this problem. These two are working in two different scenarios. We take a small look on these two technologies.

\section{D2D communication}

In LTA network data transmission will take through base station only in conventional model even for very short distance.D2D communication is wireless communication techniques makes communication between two end users without transmitting the data to the Base Station (BS)[1]. It is applicable for short range communication. Dual-hop communication process is avoided and single-hop communication has been used over here. It can be performed through licensed spectrum and unlicensed spectrum too[2]. It improves the system capacity, reliability and spectrum utilization. It is falls under four categories as follows[3]

Operator control-based relay: Relay operation between nearby device to reach the far target device is performed under the control of base station.

Device control-based relaying: Relay operation between nearby device to reach the far target device is performed under the control of base station.

Operator control-based direct communication: Direct transmission between two Device is performed under the control of base station.

Device control-based direct communication: Direct transmission between two Device is performed under the control of device itself.

\section{Cognitive Radio Technology}

Cognitive radio technology is a powerful methodology to solve the spectrum scarcity problem for the unlicensed spectrum users[4]. Most of the electromagnetic radio spectrum has be allocated for the licensed users such as TV, Airtel, jio and etc. Every little bit portion of the electromagnetic radio spectrum has been left open for the unlicensed users such as Wi-Fi, Bluetooth, and etc. [5]. Now a day's huge amount of unlicensed accessing devices is introduced. So, spectrum scarcity problem is becoming a major issue in this wireless network [8].

To overcome this problem cognitive radio technology has been introduced. Most of the TV licensed spectrums are not used or underutilized [9]. The unused licensed spectrum is called as white space or spectrum hole. Dynamic spectrum allocation is a new technique in which the spectrum holes can be accessed by unlicensed users and it will move to the next spectrum hole when the licensed spectrum users need the service [10]. The licensed spectrum users are referred as primary users and unlicensed spectrum users are referred as secondary users or CR users.

\section{SYSTEM MODEL}

In this proposed system, we considered one base station (BS), N number of unlicensed access points (AP), X denotes number of unlicensed users and $\mathrm{Y}$ denotes number of D2D$\mathrm{U}$ pairs. Number of licensed white spaces channels are denoted by $\mathrm{L}$ and band width of each channel is denoted as $\mathrm{B}_{1}$. The cognitive radio technology has been used for both CR users and D2D-U pairs.

\section{Characteristics of Wi-Fi Channel Width:}

The amount of data transmission is very much dependent to the band width. Data transmission speed can be improved by increasing the channel bandwidth. Based on the IEEE $802.11 \mathrm{n}, 2.4 \mathrm{GHzand} 5 \mathrm{GHz}$ are unlicensed spectrum band that can be used by unlicensed users like Wi-Fi users and D2D-U users. We considered $2.4 \mathrm{GHz}$ spectrum band with 11 channels that each band width is $20 \mathrm{~Hz}$. When we increase the band width, then number of channels gets reduced and transmission speed can be improved. 


\section{Cognitive Radio Technology Based Spectrum Allocation for D2d-U Communication}

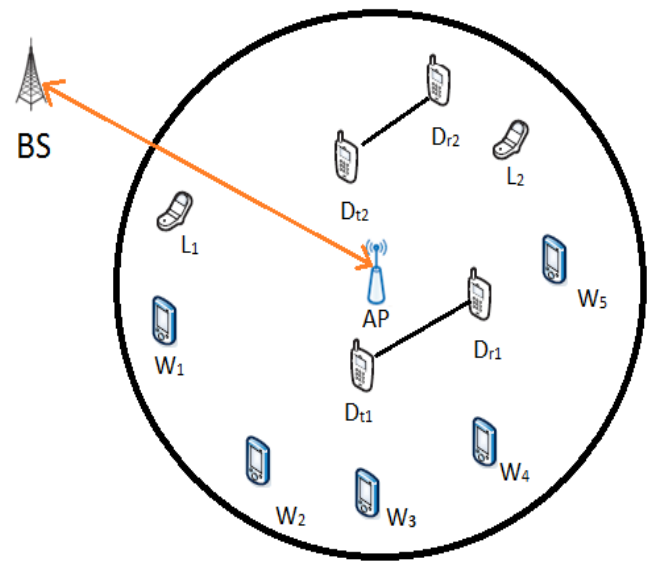

Figure1. System Model

The above statements clearly say that unlicensed spectrum users are in spectrum scarcity problem. To overcome this problem fragment Based spectrum allocation has been designed in cognitive radio technology.

\section{Fragment Based Dynamic Spectrum Allocation} Algorithm:

Case 1: Sufficient unlicensed spectrum available

Step 1: Channel request from unlicensed user to the AP.

Step 2: Allocate one channel from the available channels

Step 3: Repeat Step 1 and 2.

Case 2: Insufficient unlicensed spectrum available.

Step 1: Determine the number of CR Users

Step 2: Determine the number of D2D-U Users.

Step 3: Determine the available white spaces.

Step 4: Divide the available licensed spectrum white spaces into two portions such as $\chi$ for Wi-Fi Users and $\psi$ D2D-U users.

Step 5: Allocate the channels for CR users with in the range of $\chi$.

Step 6:Allocate the channels for D2D-U users with in the range of $\psi$.

Step 7: stop

\section{Data rate}

Data rate for the D2D- $\mathrm{U}$ is denoted as $R_{D}$ and the formula is

$R_{D}=B_{l} \log _{2}\left(1+\gamma_{n}^{d}\right)$ $-1$

Here SINR value is calculated as given for D2D-U users $\gamma_{n}^{d}=\frac{\varphi_{n P_{d}}}{I+\sum_{0}^{n} \varphi_{n P_{d}}}$ $-2$

Data rate for the $\mathrm{CR}$ is denoted as $R_{D}$ and the formula is $R_{C}=B_{l} \log _{2}\left(1+\gamma_{n}^{c}\right)$ $-3$

Here SINR value is calculated as given for CR users $\gamma_{n}^{c}=\frac{\varphi_{n P_{c}}}{I+\sum_{0}^{n} \varphi_{n P_{c}}}$

\section{Pseudo code}

Let us take number of $C R$ Users is $\boldsymbol{W}_{n}$, number of $D 2 D$ $U$ is $\boldsymbol{D}_{n}$, available licensed bandwidth is $\boldsymbol{B W}_{\boldsymbol{t}}$.

Begin

$$
\begin{aligned}
& \boldsymbol{U}_{n}=\boldsymbol{W}_{n}+\boldsymbol{D}_{n} \\
& W_{n p}=\left(\frac{W_{n}}{U_{n}}\right) * 100 \\
& D_{n p}=\left(\frac{D_{n}}{U_{n}}\right) * 100 \\
& \text { If } N C_{u}<N_{u} \\
& \text { begin }
\end{aligned}
$$

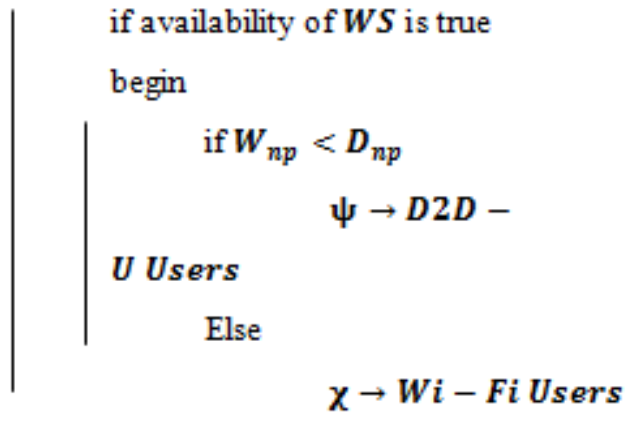

End

\section{End}

End

\section{PERFORMANCE ANALYSIS}

Here, the efficient of the proposed system has been analysed with the random spectrum allocation. In the proposed system D2D-U users will not create interference to the $\mathrm{Cr}$ users and CR users will not create interference to the D2D$\mathrm{U}$. Because the available white space range bandwidth is divided in to two separate ranges. Each user from D2D-U are accessing the channel within the rage and avoids the white space spectrum for out of range. This is same for CR users also. So interference SINR value will be reduced for both D2D-U and CR user and it leads to improvement in system sum rate.

Table1. Parameters used in simulation

\begin{tabular}{|c|c|}
\hline Parameters & Values \\
\hline D2D-U Radius & $20 \mathrm{~meter}$ \\
\hline D2D-U transmit power $P^{d}$ & $11 \mathrm{dBm}$ \\
\hline Wi-Fi user transmit power & $20 \mathrm{dBm}$ \\
\hline Channel Bandwidth & $180 \mathrm{kHz}$ \\
\hline Noise & $5 \mathrm{~dB}$ \\
\hline
\end{tabular}


The Table 1 shows that the parameter used in the proposed work.

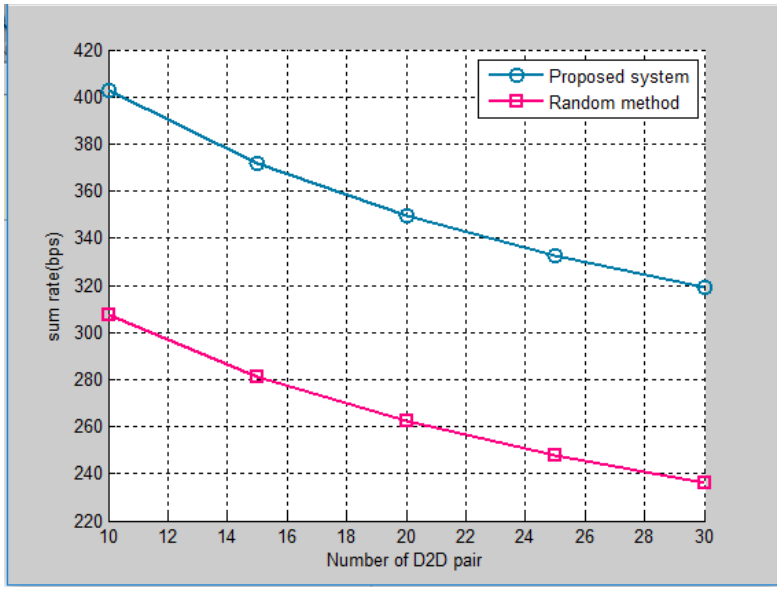

Figure2. Sum rate Vs Number of D2D-U users

The figure 2 shows that the simulations result of sum rate when the number of D2D-U users increased. The proposed system is compared with existing system (Random spectrum allocation). Obviously, when the number of users increases the sum rate will get reduced but proposed system achieved better result than the random spectrum allocation method.

\section{CONCLUSION AND FUTURE WORK}

In this paper, first described the complete system model consists of D2D-U users and CR users. The proposed algorithm differs from all other spectrum allocation technique. Here white spaces are not selected randomly for D2D-U users and CR users. Based on the number of user who needed white spaces, the available white space is divided into two ranges. One range is reserved for the D2D$\mathrm{U}$ users and another range for the CR users. For D2D-U users, channels are allocated within the reserved range and they can't access the channel from the CR user's white space range. System sum rate are determined for the proposed system and the random allocation system also. The simulation result shows that the proposed system achieved better sum rate than the random selection method. In future work interference rage among the D2D-U will be considered to determine the sum rate.

\section{REFERENCES}

1. Haykin S, 2005, "Cognitive radio: Brain-empowered wireless communications", IEEE Journal on Selected Areas in Communications, Vol-23(2),PP- 201-220.

2. Yu G, Xu L, Feng D, Yin R and Jiang J, 2014, "Joint mode selection and resource allocation for device-to-device communications," IEEE Trans. Commun., Vol-62( 11),PP- 3814-3824.

3. Feng D, Lu L, Yi Y W, Li G, Feng, D and Li S, 2013 "Device-todevice communications underlaying cellular networks," IEEE Trans. Communication. Vol-61, no. 8, PP-3541-355.

4. Tan Z, Li X, Yu F R, Chen L, Ji H, and Leung V C,2017, "Joint access selection and resource allocation in cache-enabled hons with d2d communications," in IEEE Wireless Communications and Networking Conference (WCNC), San Francisco, CA, USA, Mar. PP19-22.

5. Akyildiz I F, Lee W Y, Vuran M C, and Mohanty S,2008 "A survey on spectrum management in cognitive radio networks," IEEE Commun. Mag., Vol-46(4), PP-40-4.

6. Noura, Mahda, and Rosdiadee Nordin.2016, "A Survey on Interference Management for Device-to-Device (D2D) Communication and its Challenges in 5G Networks." Journal of Network and Computer Applications.
7. Liu R, Yu G, Qu F, and Zhang Z,2016, "Device-to-Device Communications in Unlicensed Spectrum: Mode Selection andResource Allocation", IEEE Access, Vol-4, PP-4720-4729.

8. Veeramakali, T, Jayashri, S, Prabu, S,2017 "Intelligent dynamic spectrum allocation with bandwidth flexibility in cognitive radio network. Cluster. Computing. Vol-20(2), PP-1575-158.

9. Vimal, S, Kalaivani, L, 2016,"Secure Authentication mechanisms to mitigate PUE attacks for mobile Cognitive Radio networks", IEEE CSI,IEEE-ITS \& BRNS sponsored International Conference on Computing Technologies and Intelligent Data Engineering.

10. Elhachmi J and Guennoun Z, 2016, "Cognitive radio spectrum allocation using genetic algorithm", Wireless Communication Networks Vol-1,PP-133-149.

11. Arnous R, El-Desouky A, Sarhan A. et al.,2018, "ILFCS: an intelligent learning fuzzy-based channel selection framework for cognitive radio networks" Wireless Communication Network https://doi.org/ 10.1186/s13638-018-1265-4.

12. Chen, Xu, et al.,2015, "Exploiting social ties for cooperative D2D communications: a mobile social networking case." Networking, IEEE/ACM Transactions on23.5,PP- 1471-1484.

13. Radwan, Ayman, and Jonathan Rodriguez,2015, Energy Efficient Smart Phones for 5 G Networks. Springer PP-123-135.

14. Tang, Huan, Zhi Ding, and Bernard C. Levy.2014, "Enabling D2D Communications Through Neighbor Discovery in LTE Cellular Networks." Signal Processing, IEEE Transactions on 62.19,PP5157-5170.

15. L. Lei et al., 2012,"Operator Controlled Device-to-Device Communications in LTE-Advanced Networks," IEEE Wireless Commun., vol. 19, no. 3, PP- 96-104.

16. Feng, Daquan, et al.,2014,"Device-to-device communications in cellular networks." Communications Magazine, IEEE 52.4, PP-49-55.

17. Phunchongharn, Phond, Ekram Hossain, and Dong In Kim.2013, "Resource allocation for device-to-device communications underlaying LTE-advanced networks." Wireless Communications, IEEE 20.4, PP- 91-100. 